\title{
Smart Attendance System using Face Recognition and RFID Technology
}

\author{
P. Vignesh Kanna ${ }^{1}$, Dr. K.V. Anusuya ${ }^{2}$, P. Vaishnavi ${ }^{3}$ \\ \{181159@psgtech.ac.in ${ }^{1}$, kva.ece@psgtech.ac.in ${ }^{2}, 181157 @$ psgtech.ac.in $\left.{ }^{3}\right\}$ \\ Associate Professor ${ }^{2}$, Department of ECE, PSG College of Technology, Coimbatore.,
}

\begin{abstract}
RFID, Fingerprint identification, and Iris identification techniques are used to register attendance in industries and organizations. Among all these personal identification strategies, face recognition consumes less time and is highly efficient. It has several applications in attendance management systems and security systems. In this work, a system is implemented that takes attendance for students and staff during their entry into classes or campus, employees in industries, etc, using RFID and face recognition technology. A time period can be set for taking attendance using the dashboard application which is developed using android and the database is automatically uploaded into the webserver through internet connectivity. This process is done without any human intervention. In this system, a Raspberry Pi is installed with OpenCV library and a Camera module is connected for facial Detection and Recognition. Also, an RFID reader is connected to read and verify the person's identity. The data is stored in the Firebase Database and can be accessed through Python programming. The attendance gets updated in the database and can be viewed through the developed Android application.
\end{abstract}

Keywords: Android Application, Attendance system, AWS, Face Recognition, Firebase, Raspberry Pi, RFID.

\section{Introduction}

Face recognition is a well-developed technology that is being applied to person authentication. Challenges in face recognition include illumination, pose, facial expression, aging, hair, and glasses. Radio-Frequency Identification (RFID) tags contain electronically stored information and are used for an access control system. However, a traditional RFIDbased access control system [1] identifies people only by RFID card. Anyone presenting a registered RFID card can pass the authentication even if the user is not the cardholder. To avoid this problem, face recognition [2] is used in the RFID-based access control system.

In existing biometric systems, a person has to wait in queue for a long time to give their attendance and is a time-consuming process. Also, it is quite difficult to gather the attendance manually. Hence, the speed of the attendance management process needs improvement to avoid the crowd and consider the emergency of people. A smart attendance system using RFID and Face Detection Using Video Processing is proposed to overcome the prevailing issues. The objective is to build an Attendance Management System using RFID and Face Recognition to reduce the difficulties faced in other traditional means of attendance management systems. It is planned to detect the face from the streaming video. The detected faces from the frames are stored in Cloud. Whenever the RFID is detected, the corresponding 
source image from the database is compared and the attendance is marked for the matching faces.

\section{Literature Survey}

To address the challenges faced by the existing smart attendance systems, an IoT-based attendance system using RFID was proposed in the paper [3]. It uses an Atmega328P microcontroller to interface the MFRC522 RFID reader. To increase the efficiency of Processing, the Raspberry Pi 4 Model B board is used. RFID Technology uses electromagnetic waves to detect the Identification of tags. It replaces the Barcodes and Identification cards. With this identification, attendance can be captured and updated in the MySQLite database, which is a category of SQL database. But it takes a certain time to access the data for the given queries compared to NoSQL databases. To get data synchronization and a responsive database, the Firebase Real-time database is used to quickly access the data in real-time applications. However, the disadvantages of RFID include the misuse of individual RFID tags in the absence of the respective person, easy hacking of the system, and less detection rate during the mismatch of RFID reader and tags. Fingerprint Recognition and Iris Recognition are the better solutions in the above case. But it is quite difficult to capture accurately in a fraction of seconds. These difficulties and disadvantages are overcome by Face recognition, which plays a vital role in modern smart systems. Paper [4] proposed an attendance system using face recognition. It recognizes a person's identification with a less false rate. It uses the Haar cascade algorithm to detect the faces and stores them in the MySQL database which can be seen by the administrator alone. Moreover, to verify the individual's attendance, a mobile application that can be accessed by administrators and users needs to be developed.

\section{Proposed Design}

The proposed smart attendance system shown in Fig. 1 contains a Raspberry Pi 4 Model B Board, RFID Reader, Camera, Database, and Cloud. Also, an exclusive Mobile Application is developed. Raspberry Pi 4 Board acts as the controller to monitor the Smart Attendance system with the help of other peripherals. The working principle of the system is shown in Fig. 2.

In this system, video processing and RFID detection are done during the scheduled time. Once the scheduled start time is reached, video streaming takes place as well as RFID reader enters into a reading state to detect the RFID tags. The camera generates 30 frames per second. These frames are added up for a second and the image per second is generated. It is processed using Amazon recognition API and the faces are recognized. The detected faces are stored in a collection (folder) in the AWS cloud. This process continues for every second until the end time is reached. On the other hand, once the RFID is detected, it searches for availability in the database. If RFID is matched with the database, it takes the identity photo from the database and verifies it with the detected faces from AWS collection. If there is a face match with a similarity score above 85 , attendance will be marked as "Y" in the database. This Process continues repeatedly until the system reaches the end time. Once the end time is reached, the system stops RFID detection and face recognition. The system then marks " $\mathrm{N}$ " in a database for the user IDs which are absent (not detected). The time for taking attendance and 
maintenance of the database can be controlled by the Admin through the Android application. The Attendance details can be viewed by Admin and individual users.

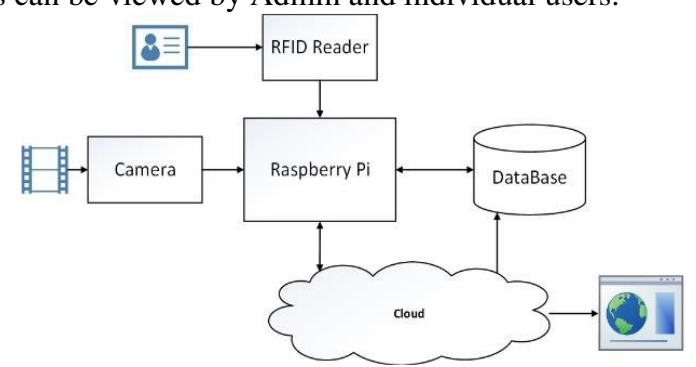

Fig. 1. Proposed Smart Attendance System

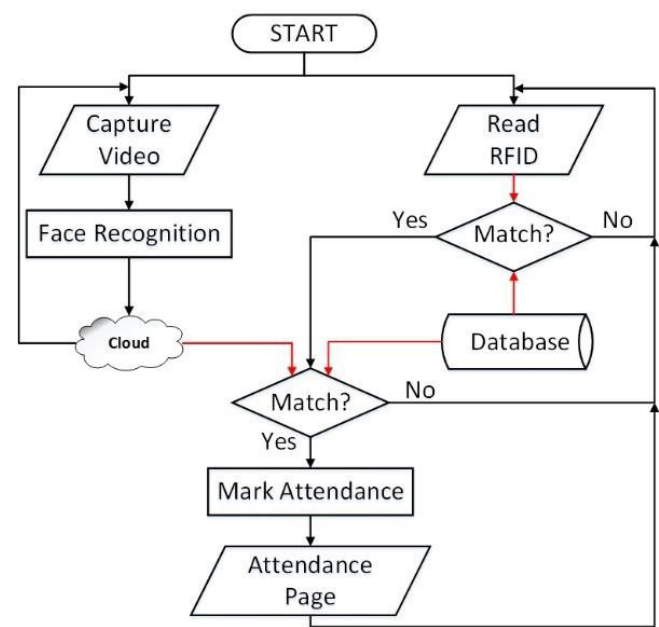

Fig. 2. Flowchart for proposed Smart Attendance System

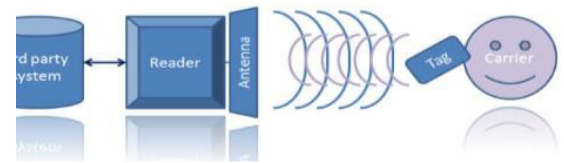

Fig. 3. Working Principle of RFID Technology

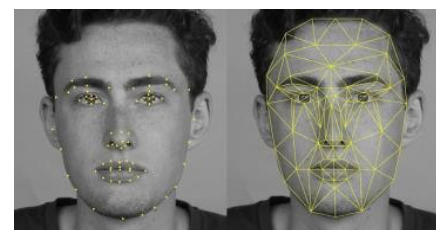

Fig. 4. Working Principle of Face detection Technique

A. RFID Detection

Passive RFID tags do not require any battery to power up the chip in the tag. Instead, the Reader transmits the RF signals which are detected by the tag as shown in Fig. 3. These RF 
signals induce current into the tag's antenna which is then used to power up the chip. Then the tag responds with the data in the chip through the coiled antenna that is detected by the Reader and transfers the data to Raspberry pi.

B. Face Detection

In Face detection, the faces are matched based on their visual geometry. Certain features like eye Left, eye Right, nose, mouth Left, and mouth Right are detected as shown in Fig. 4. A Bounding box is created when the images are analyzed. In this paper, Amazon Rekognition is used for Face detection and further Comparison.

\section{Hardware Components}

The following hardware boards and peripherals are used in the design of the proposed system.

\section{A. MFRC522 RFID Module}

The RC522 is an RF Module [5] having an RFID reader, an RFID card, and a key chain. The module operates at $13.56 \mathrm{MHz}$ in the ISM band at a typical operating voltage of $3.3 \mathrm{~V}$. The tag has $1 \mathrm{~KB}$ memory to store the detected data. The RC522 reader module can both read and write data into these memory elements. The reader can read data only from passive tags that operate at $13.56 \mathrm{MHz}$.

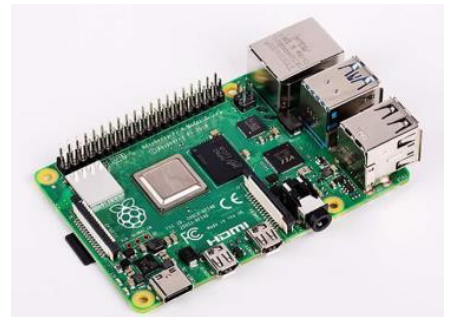

Fig. 5. Raspberry Pi 4 Model B Board

B. Raspberry Pi 4 Model B Board

Raspberry Pi 4 Model B [6] shown in Fig. 5 has exceptional increase in processor speed, memory capacity, and multimedia performance with good connectivity. Its key features include a quad-core processor, dual-display support via a pair of micro-HDMI ports, hardware video decode, RAM support up to $8 \mathrm{~GB}$, dual-band 2.4/5.0 GHz wireless LAN, Bluetooth 5.0, Gigabit Ethernet and USB 3.0.

\section{Logitech C270 HD Camera}

Logitech C270 HD camera [7] is used in the proposed design. It can take a 3MP photo (software enhanced). The webcam adjusts to poor lighting conditions automatically to produce the best possible image. The background noise won't spoil the video. It offers a high-quality resolution with reduced blur in the image.

\section{Software Tools}

The software development involves the following tools and platforms. A. Raspberry Pi OS 
Raspberry $\mathrm{Pi}$ OS is the free operating system based on Debian optimized for the Raspberry Pi hardware. Raspberry Pi OS has precompiled software bundled for easy installation on Raspberry Pi. Raspberry Pi has a graphical SD card writing tool that works on Mac OS, Ubuntu 18.04, and Windows, to download the image and install it automatically into the SD card.

\section{B. OpenCV Python}

OpenCV is an open-source library for computer vision, machine learning, and image processing. OpenCV supports the programming languages like Python, $\mathrm{C}++$, Java, etc. It can process images and videos to identify objects, faces, or even the handwriting of a human when it is integrated with various libraries such as NumPy - A highly optimized library for numerical operations. Out of the number of pixel operations supported, the dyadic operator which takes two inputs $f_{0}(x)$ and $f_{1}(x)$ called the linear blend operator is used in the design.

$(x)=(1-\alpha)_{0}(x)+\alpha f_{1}(x)$

The operator given by (1) adds up two frames into a single frame, which in turn removes the blur caused concerning time. By varying $\alpha$ from 0 to 1 , this operator can be used to perform a temporal cross- dissolve between the two images or videos.

C. Amazon Rekognition API

Amazon Rekognition is a service for image analysis. Using this service, detection of objects, scenes, text, faces or even recognition of celebrities and identification of inappropriate content in images can be built. It can compare one face in the source image with up to 15 detected faces in the target image. It is a cloud-managed service that has different SDKs for programming languages. It can also be used to search and compare faces. Its API enables the quick addition of deep learning-based visual search and image classification to the proposed design. The boto3 library file [8] provided by Amazon Web Services and face detection function are used in the proposed design.

\section{Firebase Realtime Database}

The Firebase Realtime Database allows securing access to the database directly from client-side code. Data is persisted locally, and even while offline, real-time events continue to fire, giving the end-user a responsive experience. When the device regains connection, the Realtime Database synchronizes the local data changes with the remote updates that occurred while the client was offline, merging any conflicts automatically. When integrated with Firebase Authentication, the developers can define the accessing person with the data and the access method. The Firebase Realtime Database API [9] is designed to only allow operations that can be executed quickly.

\section{Android Application}

An Android application named "SAS" is developed to view the database details and its attendance updation. It can be edited and controlled by the admin. Fig. 6 displays the Home and admin page with a few options like Add User, Edit, Attendance, Schedule and Log out. Using ADD USER option, the admin can include the user's information such as User Name, RFID, E-mail, Password, and the User status as Staff / Student.

The EDIT USER option in Fig.7 enables the admin to edit the User profile in some situations when the user loses his/her ID or when there is some problem with the Reader.

The SCHEDULER page in Fig. 8 schedules the time at which RFID detection and Face recognition takes place in 


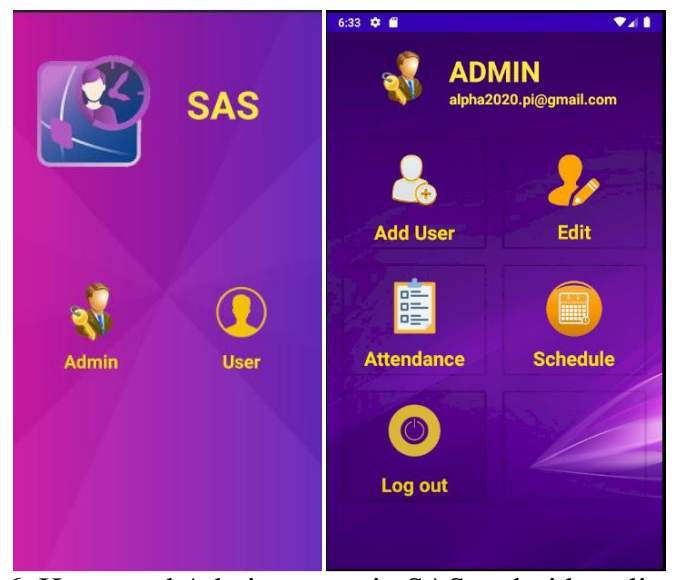

Fig. 6. Home and Admin screen in SAS android application

Raspberry $\mathrm{Pi}$ in a day or week. The attendance of the user along with the details is displayed for the selected ID through the spinner box.

The attendance for an individual user can be seen by signing in to the Android application with their respective login credentials as shown in Fig. 9.

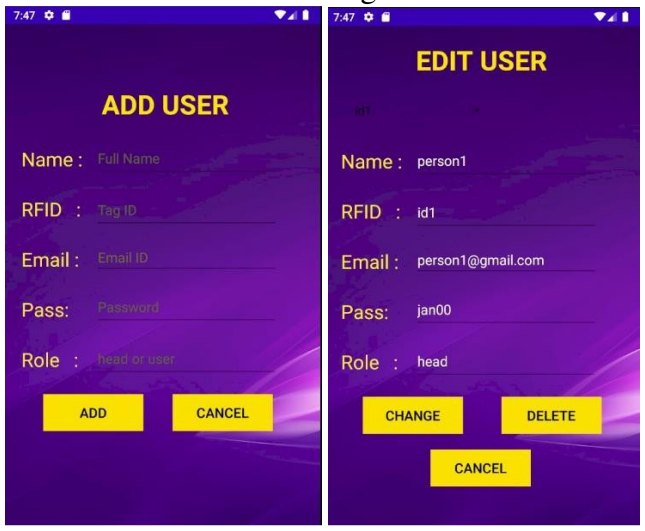

Fig. 7. Add user and edit user screen 


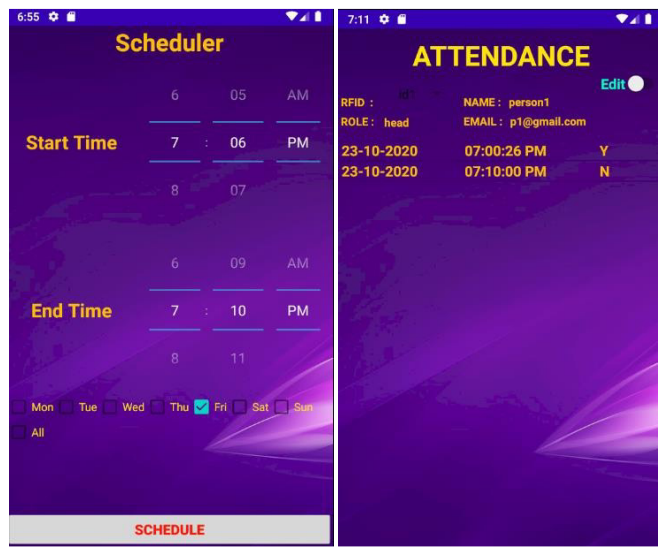

Fig. 8. Scheduler and attendance view screen

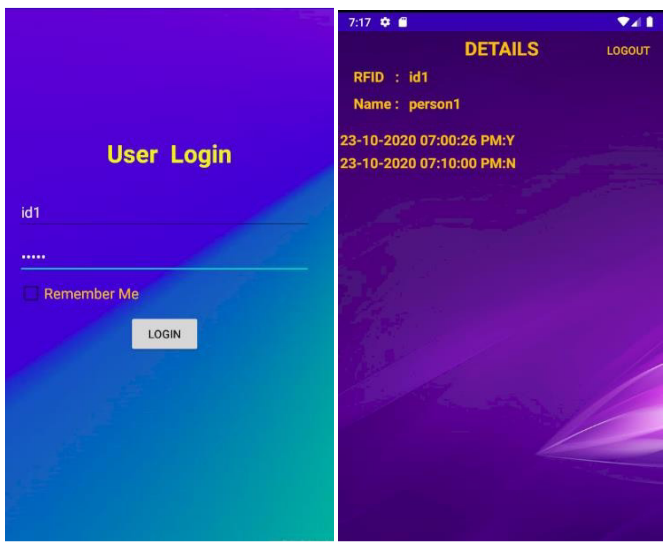

Fig. 9. User Login page and user's attendance view screen

\section{Firebase Realtime Database}

In this paper, the Firebase database is used to store the user details, attendance, and schedule of Raspberry Pi. Three database keys are used in the design and are listed below. User's key stores the user's profile information such as email, name, password, RFID, and role from adding user option in the android application as shown in Fig. 10.

Fig. 11 shows the Schedule key stored in the database from the Android application. When the scheduling starts, the system starts detecting the RFID and face recognition simultaneously. When the scheduling time reaches the end, the above two processes are turned off, and again when the Start time of the schedule commences, the processes are repeated based on the schedule.

Once the RFID is detected, the attendance gets updated in the Attendance key in the Firebase database based on three conditions. Attendance gets updated as: 
- $\quad$ Y - when Both RFID and Detected faces are matched with the User database.

- R- when only RFID is matched with the user database and face not detected.

- $\quad \mathrm{N}$ - when both RFID and face are not detected at the end of schedule time.

The working of Real-Time Database in the Google Firebase is checked using pyre-base library file [10] in Python. The firebase database is also connected with an android application for the user interface. Fig. 12(a) displays the attendance table when the first schedule is completed. When the schedule reaches its end time, the system marks $\mathrm{N}$ (indicating that the user is absent) for the IDs that are not detected and R (indicating that only RFID is detected and face does not match) for the IDs where faces are not matched. Fig. 12(b) shows the attendance table after the second schedule is completed. In the second schedule, ID2 is detected; but the face is not matched. Hence it is marked as R.

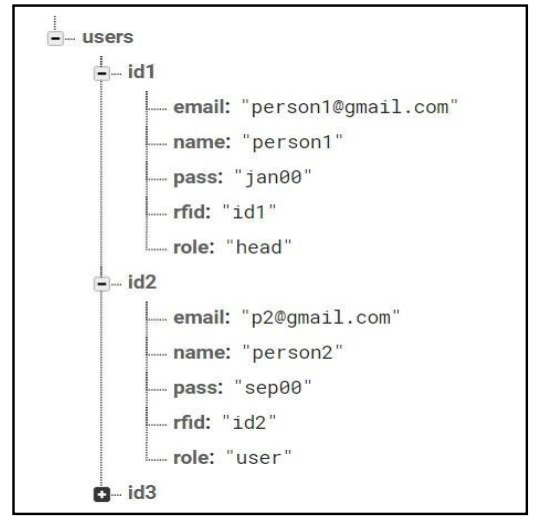

Fig. 10. User's information stored in Firebase Real time database

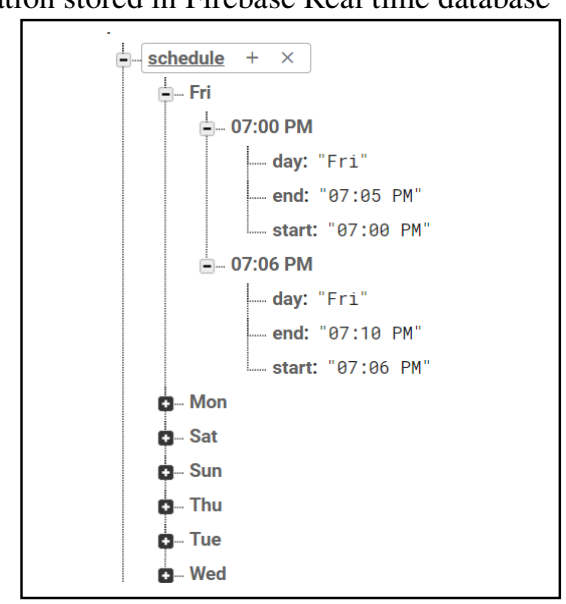

Fig. 11. Working time in the schedule key of Raspberry Pi 


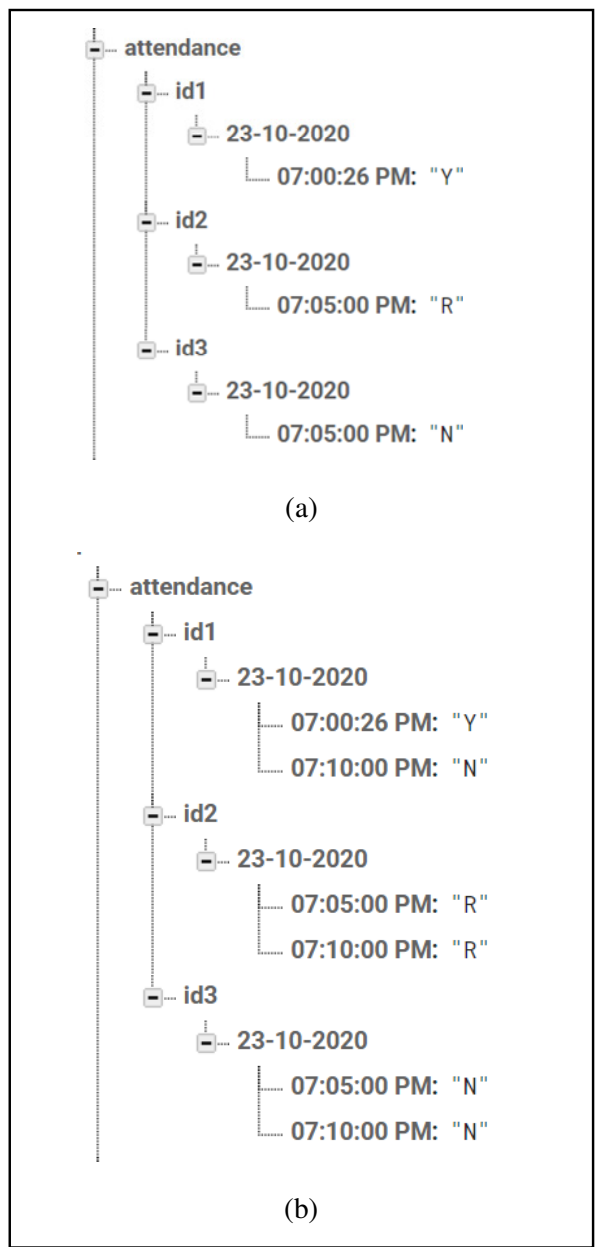

Fig. 12. Attendance key-pair values at the end of the first schedule and second schedule

\section{Results}

The RFID Module and the camera are interfaced [11] with the Raspberry Pi as shown in Fig. 13. The working of the smart attendance system programmed using Python is constructed and its performance is tested.

The scheduler page displays the scheduled time for the final smart attendance system from the android application. It is shown in Fig. 14. 
Fig. 15 shows the Schedule time for the working of face detection and RFID detection which is stored in the

firebase realtime database.

Fig. 16 shows the user's key-value pairs in a database which contains the IDs and the user's profile details that were used during the system verification

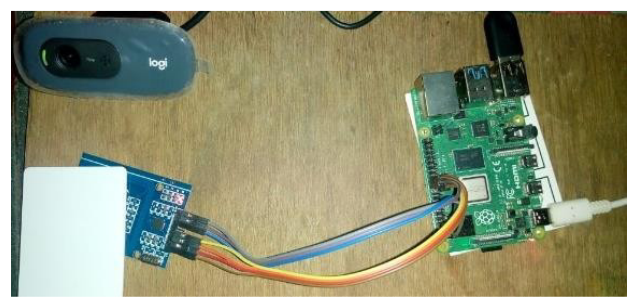

Fig. 13. Interfacing of Raspberry Pi with RFID and USB Camera Module

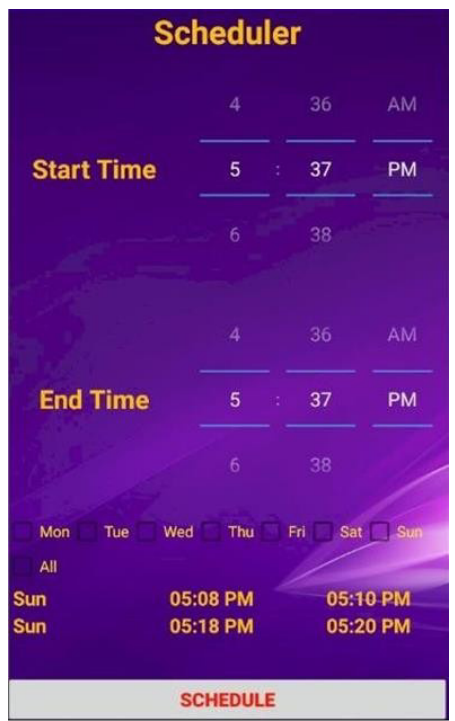

Fig. 14. Scheduler screen during scheduling the attendance monitoring time. 


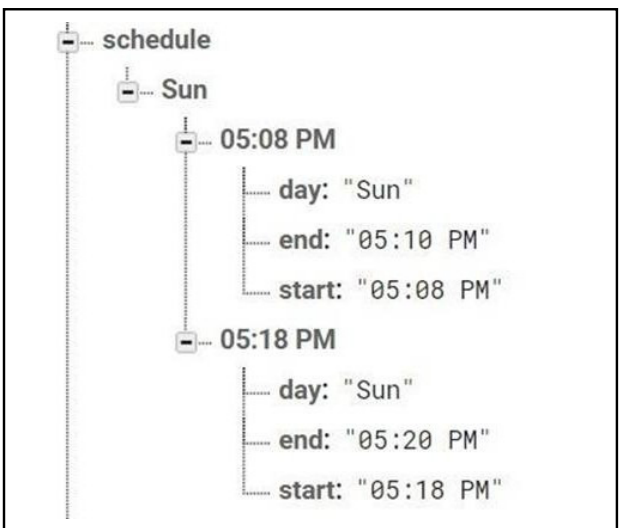

Fig. 15. Schedule time stored in the database -... users<smiles>C1=[GeH]CC[Al]1</smiles>

- - id1

_.. email: "p1@gmail.com"

-name: "person1"

-wass: "janø0"

L.. rfid: "id1"

r... role: "head"

- - id2

L. email: "p2@gmail.com"

-name: "person2"

-wass: "feber"

- rfid: "id2"

role: "user"

$-\ldots$ id3

L... email: "p3@gmail.com"

-name: "person3"

-- pass: "mare0"

Fig. 16. Users details stored in the database 


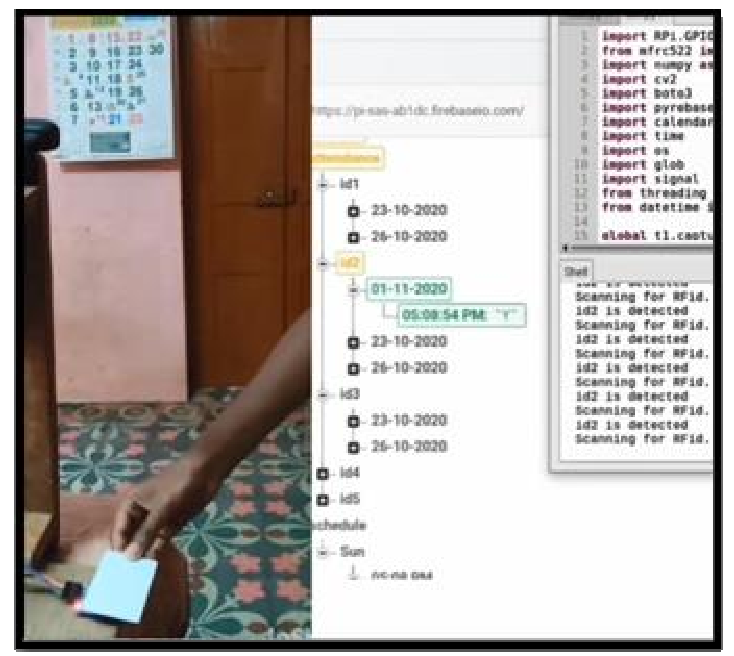

Fig. 17. Smart attendance system verification.

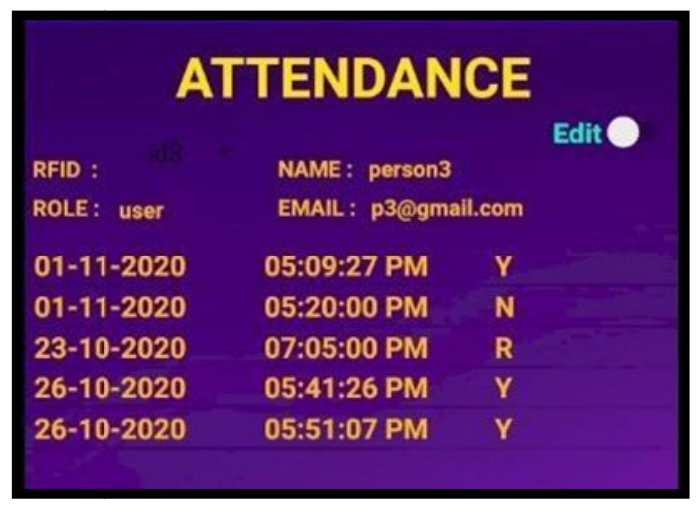

Fig. 18. Attendance view in Android application

The implementation of our smart attendance system is done with few students and the proper functioning of our system is also verified which is shown below in Fig. 17.

The attendance is recorded and stored in the Database. The recorded attendance can be viewed by the administrator as well as by the users from their respective pages in the android application as shown in Fig. 18.

\section{Conclusion}

The proposed, Smart Attendance System using RFID and Face Recognition is an effective solution for attendance and verification systems used in any organization with many 
employees and definitely at where the speed of the attendance process is a limitation. This integrated solution of both ideas can also increase the efficiency of verification since there are two levels of security during the process of verification. In the future, this system can be enhanced by improving the RFID read range over 3 meters using UHF RFID Readers, increasing the provisions and options for users in mobile applications, developing a web page that is compatible with all platforms like Desktop, PC, Mobile, etc. and making the system compatible with double way face detection using the rotatable camera.

\section{References}

[1] U. Koppikar, S. Hiremath, A. Shiralkar, A. Rajoor, and V. P. Baligar, "IoT Based Smart Attendance Monitoring System using RFID," 2019 1st International Conference on Advances in Information Technology (ICAIT), Chikmagalur, India, 2019, pp. 193-197, doi: 10.1109/ICAIT47043.2019.8987434.

[2] S. Hasban et al., "Face recognition for Student Attendance using Raspberry Pi," 2019 IEEE AsiaPacific Conference on Applied Electromagnetics (APACE), Melacca, Malaysia, 2019, pp. 1-5, doi: 10.1109/APACE47377.2019.9020758.

[3] R. Kiran Kumar and S Mekala, "Face Recognition attendance system using Raspberry Pi", International

[4] Journal of Pure and Applied Mathematics, vol. 115, October 2018.

[5] S. N. Shah and A. Abuzneid, "IoT Based Smart Attendance System (SAS) Using RFID," 2019 IEEE Long Island Systems, Applications, and Technology Conference (LISAT), Farmingdale, NY, USA, 2019, pp. 1-6, doi: 10.1109/LISAT.2019.8817339.

[6] RFID MFRC522 module datasheet Anline]. Available: https://www.nxp.com/docs/en/data- sheet/MFRC522.pdf

[7] Raspberry pi 4 Model $\mathrm{B}$ datasheet [Online] Available: https://www.raspberrypi.org/documentation/hardwa re/raspberrypi/bcm2711/rpi_DATA_2711_1p 0_preliminary.pdf

[8] Logitech C270 HD camera datasheet [Online]. Available: http://www.farnell.com/datasheets/1746965.pdf

[9] Boto3 Documentation (AWS-CLI). [Online]. Available: https://boto3.amazonaws.com/v1/documentation/api

[10] /latest/index.html

[11] Firebase Documentation for Android applications. [Online]. Available: https://firebase.google.com/docs/database/android

[12] Pyrebase library Documentation [Online]. Available: https://github.com/thisbejim/Pyrebase/blob/master/ README.md

[13] Setup Raspberry pi with RFID RC522 Chip [Online]. Available: https://pimylifeup.com/raspberrypi- RFID-rc522/ 